Short Communication

\title{
A challenging diagnosis of reversible "vascular" dementia: Cerebral amyloid angiopathy-related inflammation
}

\author{
L. Poli ${ }^{\mathrm{a}, *}$, V. De Giuli ${ }^{\mathrm{b}}$, F. Piazza ${ }^{\mathrm{c}}$, I. Volonghi ${ }^{\mathrm{a}}$, G. Bigliardi ${ }^{\mathrm{e}}$, S. Vallone ${ }^{g}$, P.F. Nichelli ${ }^{\mathrm{e}}$, \\ R. Gasparotti ${ }^{\mathrm{d}}$, A. Zinif ${ }^{\mathrm{f}}$, A. Padovani ${ }^{\mathrm{b}}$, A. Pezzini ${ }^{\mathrm{b}}$

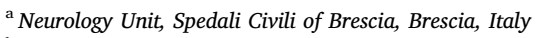 \\ ${ }^{\mathrm{b}}$ Department of Clinical and Experimental Sciences, Neurology Clinic, University of Brescia, Brescia, Italy \\ ${ }^{\mathrm{c}}$ School of Medicine, University of Milano - Bicocca, Monza, Italy \\ ${ }^{\mathrm{d}}$ Department of Medicine and Surgery, Radiology and Public Health, University of Brescia, Brescia, Italy \\ e Department of Neuroscience, Neurology Clinic, Stroke Unit, University of Modena and Reggio Emilia, Nuovo Ospedale Civile "S.Agostino-Estense", AUSL Modena, Italy \\ ${ }_{\mathrm{f}}^{\mathrm{f}}$ IRCCS Istituto di Scienze Neurologiche di Bologna, UOC Neurologia e Rete Stroke metropolitana, Ospedale Maggiore, Bologna, Italy \\ ${ }^{\mathrm{g}}$ Department of Neuroradiology, Nuovo Ospedale Civile "S.Agostino-Estense", AUSL Modena, Modena, Italy
}

\section{A B S T R A C T}

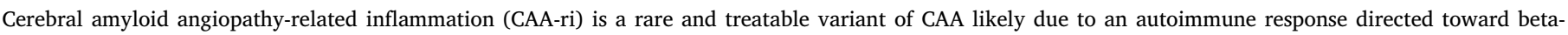

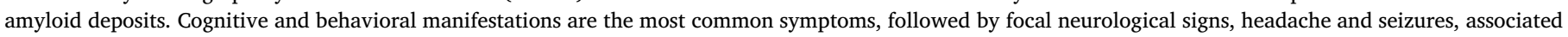
with characteristics neuroradiological features on brain magnetic resonance imaging (MRI).

We describe the clinical course, radiological features and therapeutic approach of two patients with probable CAA-ri with the aim of emphasizing the importance of an early diagnosis of this potentially reversible disease in different neurological settings, such as memory clinics and stroke units.

\section{Introduction}

Cerebral amyloid angiopathy (CAA), the most common cause of lobar intracerebral hemorrhage in the elderly, is the final result of the deposition of $\beta$-amyloid in the wall of small to medium sized arteries, and less frequently veins and capillaries, of the leptomeninges and brain (Wermer and Greenberg, 2018; Greenberg and Charidimou, 2018). Cerebral amyloid angiopathy-related inflammation (CAA-ri) is a rare and relatively unknown feature of CAA. It is characterized by a variable combination of clinical manifestations, such as subacute confusion, cognitive decline, headache, epileptic seizures and focal neurological deficits (Eng et al., 2004), associated with characteristic neuroradiological features on brain magnetic resonance imaging (MRI) (Auriel et al., 2016; Piazza et al., 2013). As suggested by the neuropathological evidence of vascular and perivascular inflammation, these features are thought to be the result of an autoantibody-mediated inflammatory response directed toward $\beta$ amyloid (A $\beta$ ) deposited in the walls of cerebral blood vessels (Piazza et al., 2013; DiFrancesco et al., 2011).

The observation of clinical and radiological improvement of CAA-ri after administration of high dose corticosteroids or other immunosuppressive agents emphasizes the importance of making a correct and prompt diagnosis in clinical practice.

In the present report, we illustrate the clinical presentation, neuroimaging features, and outcome of two patients with probable CAAri (according to Auriel et al. criteria) (Auriel et al., 2016) who received immunosuppressive therapy.

\section{Case 1}

A 79-year-old male was admitted to the neurological ward because of rapidly-progressive neurobehavioral symptoms (aggressiveness, agitation and confusion) and gait unsteadiness, responsible for his fall and consequent head trauma. His past medical history included systemic arterial hypertension and acute intracerebral hemorrhage (ICH) involving the left temporal lobe one year before, in association with MRI evidence of cortical-subcortical microbleeds and superficial siderosis (Fig. 1, Panel A), as well as a 6-month history of slight memory deficits and worsening of pre-existing language impairment. Because the cerebral hematoma was not surgically evacuated at the time of occurrence, we made the diagnosis of probable CAA according to the modified Boston criteria (Greenberg and Charidimou, 2018), after exclusion of secondary causes of ICH.

On admission, the neurological examination evidenced spatial and temporal disorientation, psychomotor slowness, and confusion. The Mini-Mental State Examination (MMSE) score was 8/30. Brain MRI showed bilateral T2 and fluid-attenuated inversion recovery (FLAIR)

\footnotetext{
* Corresponding author at: Neurology Unit, Spedali Civili, P.le Spedali Civili, 1, 25123 Brescia, Italy.

E-mail address: loris.poli@gmail.com (L. Poli).
} 
Panel A

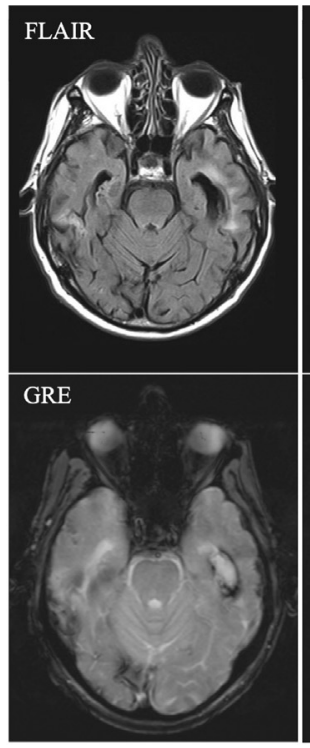

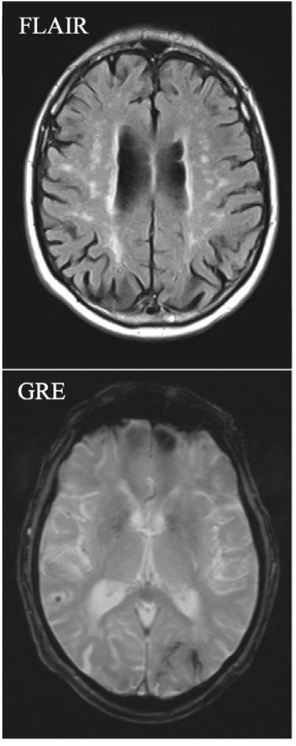
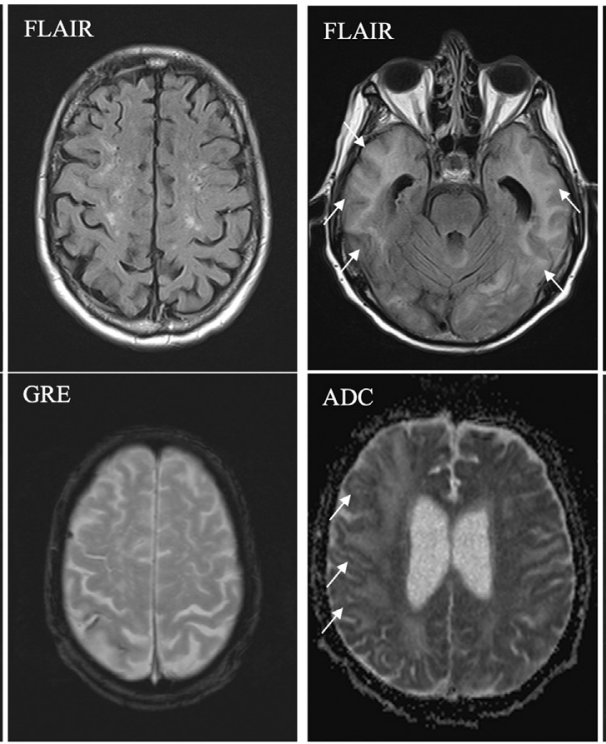

Panel B

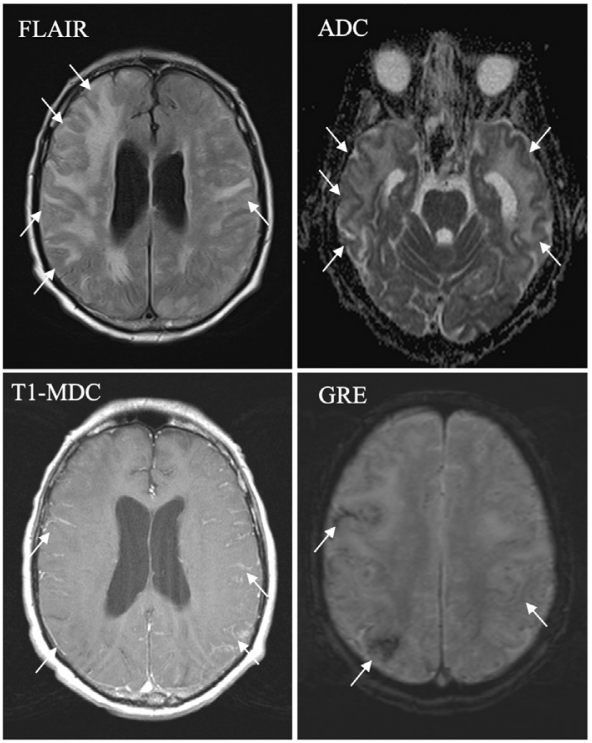

Fig. 1. Brain MRI, case 1 .

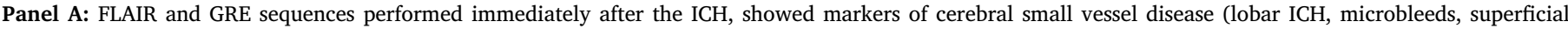
siderosis), consistent with probable CAA.

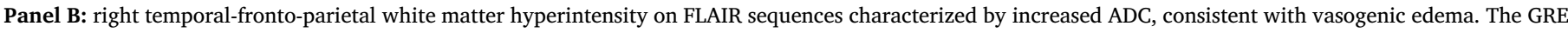

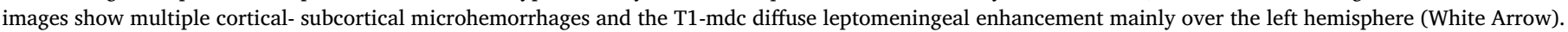

subcortical confluent hyperintensities with greater representation in the right fronto-parietal lobe, characterized by increased apparent diffusion coefficient, consistent with vasogenic edema. T2\%-weighted gradientecho (GRE) sequences showed cortical micro-hemorrhages and right fronto-parietal cortical hemosiderosis, with diffuse leptomeningeal enhancement, mainly over the left hemisphere, after gadolinium administration (Fig. 1, Panel B). Blood tests including erythrocyte sedimentation rate, C-reactive protein, serum protein electrophoresis, angiotensin-converting enzyme, and adenosine deaminase were normal. Cerebrospinal fuid (CSF) examination showed hyperproteinorrachia $(371 \mathrm{mg} / \mathrm{dL}$, normal range, $<45 \mathrm{mg} / \mathrm{dL})$ with normal glucose content and cell count. No bacteria, mycobacteria or fungi were detected on CSF cultures. Molecular analysis of the Apolipoprotein E gene (APOE) revealed apoE $\varepsilon 3 / \varepsilon 4$ genotype. The search for CSF antiamyloid $B(A \beta)$ autoantibodies proved positive (at the concentration of $193.5 \mathrm{ng} / \mathrm{mL}$ ) (Piazza et al., 2013). Based on clinical and neuroimaging findings we made the diagnosis of probable CAA-ri, according to Auriel et al. criteria (Auriel et al., 2016). Three weeks after hospital admission, intravenous corticosteroid therapy was started (methylprednisolone $1000 \mathrm{mg}$ i.v./die for the first 3 days), followed by oral administration (prednisolone $1 \mathrm{mg} \mathrm{kg} / \mathrm{die}$ ). Because of the progressive alteration of the state of consciousness in the following days, we decided to administer intravenous cyclophosphamide ( $1200 \mathrm{mg}$ at 3 -week intervals). After a transient behavioral improvement, the patient continued to deteriorate and died 40 days after symptoms onset. No postmortem examination was performed.

\section{Case 2}

A 76-year-old woman was admitted to the geriatric ward because of 5-day gait unsteadiness along with severe frontal and periorbital headache and rapidly progressive behavioral alterations (agitation and confusion). Past medical history included systemic arterial hypertension, mild depression, amnesic mild cognitive impairment and retinal detachment involving her left eye. Two days after admission she developed acute language impairment with psychomotor slowing. Brain computed tomography (CT) scan showed bilateral parietal convexity subarachnoid hemorrhage in addiction to diffuse bilateral cortico-subcortical hypodensity. Neurological examination showed non fluent aphasia, along with unsteady and wide-based gait.

Brain MRI showed left temporo-occipital white matter hyperintensity on T2/FLAIR sequences, characterized by increased apparent diffusion coefficient and hypointensity on T1-weighted sequences, consistent with vasogenic edema while GRE revealed cortical-subcortical micro-hemorrhages along with a convexity subarachnoid hemorrhage (Fig. 2, Panel A). We also detected mild leptomeningeal enhancement after gadolinium administration. To rule out either vascular anomalies or central nervous system vasculitis we performed a conventional cerebral angiography which didn't show signs consistent with those clinical hypotheses. Blood workup for infection and autoimmune markers resulted negative as well. CSF examination showed mildly elevated protein level $(65 \mathrm{mg} / \mathrm{dL}$, normal range $<50 \mathrm{mg} / \mathrm{dL})$ with normal glucose content and cell count, in absence of infection. Screening of APOE revealed $\varepsilon 3 / \varepsilon 4$ genotype. The search for CSF anti-A $\beta$ autoantibodies turned out to be positive (at the concentration of $113.75 \mathrm{ng} / \mathrm{mL}$ ) (Piazza et al., 2013). Clinical data together with neuroimaging findings were consistent with the diagnosis of probable CAAri, according to Auriel et al. criteria (Auriel et al., 2016). Corticosteroid therapy with methylprednisolone $1000 \mathrm{mg}$ i.v./daily for the first 3 days was started, followed by prednisolone $75 \mathrm{mg} /$ daily, which was gradually tapered in the following months. A few days after the therapy was started, a marked clinical improvement with resolution of confusion and speech disorder was detected, and the patient returned to her premorbid state. After 4 weeks of treatment we performed a follow up brain MRI that revealed dramatic improvement of the white matter lesions, in addition to clear reduction of the vasogenic edema. The 3month follow-up MRI showed complete resolution of the vasogenic edema (Fig. 2, Panel B) along with an evident improvement on cognitive deficits.

\section{Discussion}

CAA-ri is an aggressive, uncommon and likely underdiagnosed subtype of CAA. Although a definite diagnosis requires pathologic 
Panel A
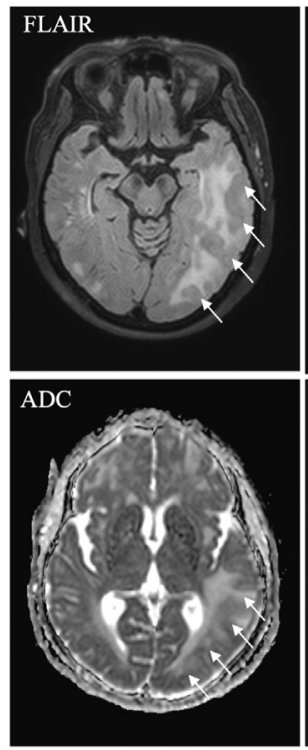
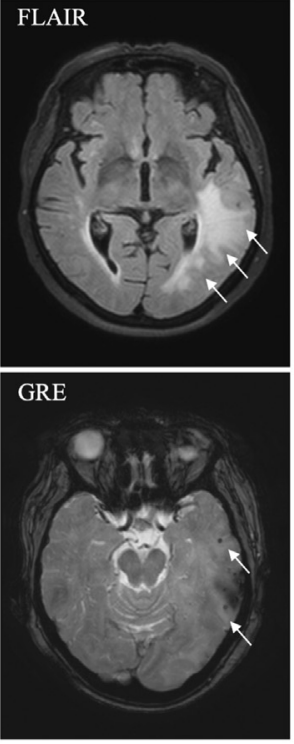
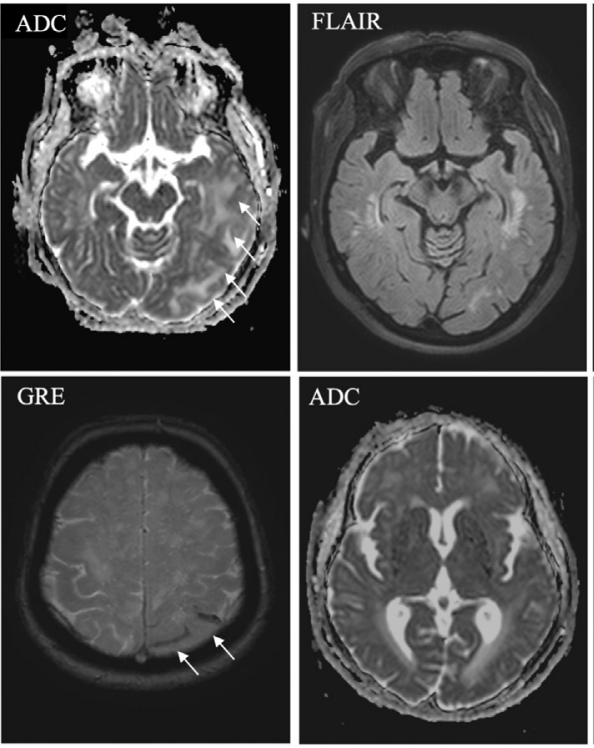

Panel B

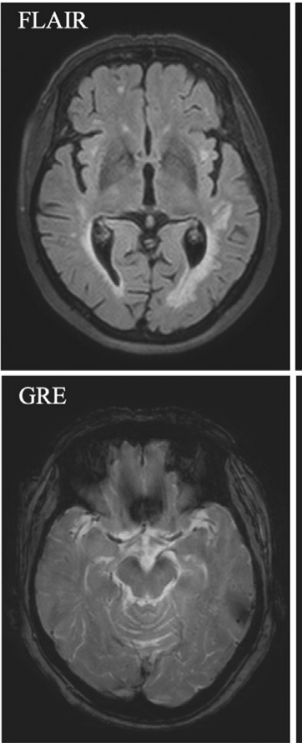

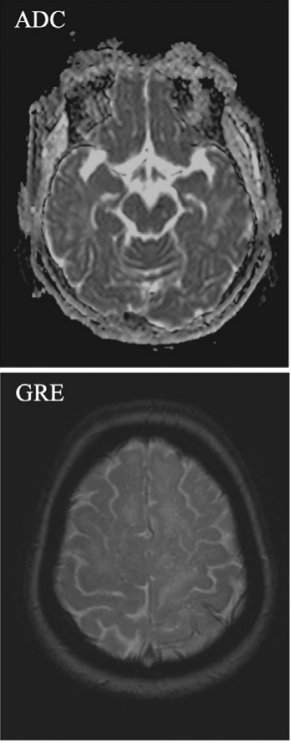

Fig. 2. Brain MRI, case 2 .

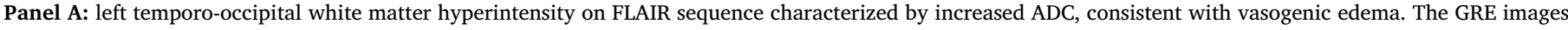
showing cortical-subcortical microhemorrhages at the same level and the T1-mdc left temporal leptomeningeal enhancement (White Arrow).

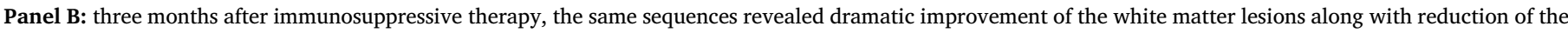
vasogenic edema.

confirmation, clinical data, MRI findings, as well as response to immunosuppressive therapy are strongly suggestive of the disease (Auriel et al., 2016). Based on histopathology of brain biopsy, two forms of CAA-ri can be distinguished: inflammatory CAA (ICAA) and Amyloid- $\beta$ Related Angiitis (ABRA). Specifically, while ICAA is characterized by a perivascular inflammation, ABRA is characterized by transmural and intramural inflammation, often with granulomas formation (Moussaddy et al., 2015; Ng et al., 2017; Chu et al., 2016). ICAA and ABRA share several clinical and neuroradiological features and are indistinguishable from each other without histopathological data (Salvarani et al., 2016; Chu et al., 2016).

Both described cases showed a clinical picture characterized by rapidly progressive behavioral changes and cognitive dysfunction. In line with the diagnostic criteria (Auriel et al., 2016), the diagnosis in the reported cases was probable CAA-ri. Detectable anti-A $\beta$ antibodies in the CSF should be considered as an additional evidence in favor of this diagnosis in both patients. Indeed, as already described by Piazza and collaborators (Piazza et al., 2013), CSF anti-A $\beta$ antibodies seem to be directly implicated in the pathogenesis of CAA-ri: anti-A $\beta$ antibody titres are very high during the acute phases of the disease and decrease upon immunosuppressive treatment, when a concurrent improvement of the clinical status occurs (DiFrancesco et al., 2011; Charidimou, 2016). Although a validation of cut-offs has not yet been performed, previous studies reported significantly higher values of CSF anti-A $\beta$ antibodies in subjects affected by CAA-ri compared to control subjects (Piazza et al., 2013), just as in our cases. Therefore, the research of antiA $\beta$ antibodies not only focuses on the possible pathogenic mechanism of the disease but also represents a possible useful biomarker for the diagnosis and non-invasive monitoring of CAA-ri (DiFrancesco et al., 2011; Moussaddy et al., 2015; Ng et al., 2017; Salvarani et al., 2016; Chu et al., 2016; Charidimou, 2016; Sakaguchi et al., 2011).

As already reported in the literature, most patients with CAA-ri show partial to complete response to high dose corticosteroids or other immunosuppressive agents associated with clinical and radiological improvement (Kirshner and Bradshaw, 2015). Some authors suggest that it may be reasonable to use empirical immunosuppressive therapy, without brain biopsy, for patients meeting the criteria proposed for probable CAA-ri (Charidimou, 2016; Sakaguchi et al., 2011; Kirshner and Bradshaw, 2015). To diagnose the disease with a high degree of certainty based only on clinical and MRI criteria would avoid an invasive brain biopsy that would aggravate the patient's discomfort and increase the risk of complications. Obviously, the lack of pathological data prevents any conclusions regarding the differential diagnosis between the two disease entities ABRA and ICAA. Notwithstanding, both forms require prompt immunosuppression and show a similar response to treatment (Auriel et al., 2016; Moussaddy et al., 2015; Ng et al., 2017).

In our patients the diagnosis of CAA-ri was made by using both the suggested diagnostic criteria (Auriel et al., 2016) and the detection of anti-A $\beta$ antibodies on CSF. Consequently, we opted for corticosteroids in both our cases. It is reasonable hypothesize that the delay in initiating the immunosuppressive therapy administration, along with the poor general conditions and the diffuse cerebral involvement, facilitated the negative outcome in the first patient, while the rapid detection of the disease and the prompt initiation of specific therapy resulted in excellent clinical outcome in the second case.

The two described cases have in common both the clinical presentation (cognitive and behavioral changes) and the neuroradiological features. Nevertheless, the first case had history of previous cerebral hemorrhage with a diagnosis of probable CAA, while in the second one the CAA-ri was the first manifestation of an underlying CAA.

Therefore, in the case of rapidly progressive cognitive decline, it is mandatory to consider CAA-ri among the possible diagnoses, even if a clinical history suggestive of the underlying CAA is lacking; this is also applicable to patients with Alzheimer's disease, even in the mild cognitive impairment phase (Boncoraglio et al., 2015; Piazza and Winblad, 2016; Carmona-Iragui et al., 2016).

In this scenario, brain MRI with specific sequences (FLAIR, GRE/ SWI and ADC) could allow the recognition of the characteristic neuroradiological features. In particular, according to the clinico-radiological criteria for the diagnosis of CAA-ri recently proposed by Auriel and colleagues (Auriel et al., 2016), both mono or bilateral white matter hyperintensities extending to the immediately subcortical white matter along with cortico-subcortical hemorrhages should be 
considered highly specific for CAA-ri (Piazza et al., 2013), with subacute cognitive decline or seizures as the predominant clinical symptoms (Kinnecom et al., 2007). As regards laboratory data, in addition to the detection of anti-A $\beta$ antibodies on CSF, the determination of the Apolipoprotein E genotype (APOE) may be considered as a further contribution to the diagnosis. Actually, although no specific genotypephenotype correlations have been established so far, the $\varepsilon 4$ allele has been consistently reported to increase the risk of developing CAA-ri (Kinnecom et al., 2007) a finding that provides further strength to this diagnosis in our cases.

In conclusion, CAA-ri is a challenging and important diagnosis to make in clinical practice, because of the potential benefits of a prompt immunosuppressive therapy (with steroids and other therapeutic agents) in interrupting the underlying biological process and reversing the clinical symptoms and neuroradiologic involvement.

\section{Funding}

This work was partially supported by Fondazione Cariplo (biomarkARIA Research Project - 2015-0820); the Alzheimer's Association (ModelCAA Research Grant - AARG-18-561699); and the SINdem-CAA Study Group.

\section{Declaration of Competing Interest}

None.

\section{References}

Auriel, E., Charidimou, A., Gurol, M.E., et al., 2016. Validation of clinicoradiological criteria for the diagnosis of cerebral amyloid angiopathy-related inflammation. JAMA Neurol. 73, 197-202.

Boncoraglio, G.B., Piazza, F., Savoiardo, M., et al., 2015. Prodromal Alzheimer's disease presenting as cerebral amyloid angiopathy-related inflammation with spontaneous amyloid-related imaging abnormalities and high cerebrospinal fluid anti-A $\beta$ autoantibodies. J. Alzheimers Dis. 45 (2), 363-367.

Carmona-Iragui, M., Fernández-Arcos, A., Alcolea, D., et al., 2016. Cerebrospinal fluid anti-amyloid- $\beta$ autoantibodies and amyloid PET in cerebral amyloid angiopathy-related inflammation. J. Alzheimers Dis. 50 (1), 1-7.

Charidimou, A., 2016. Cerebral amyloid angiopathy-related inflammation biomarkers: where are we Now? J. Alzheimers Dis. 50 (1), 9-11.

Chu, S., Xu, F., Su, Y., et al., 2016. Cerebral amyloid angiopathy (CAA)-related inflammation: comparison of inflammatory CAA and amyloid- $\beta$-related angiitis. J. Alzheimers Dis. 51 (2), 525-532.

DiFrancesco, J.C., Brioschi, M., Brighina, L., et al., 2011. Anti-A?? autoantibodies in the CSF of patient with cerebral amyloid angiopathy-related inflammation: a case report. Neurology. 76, 842-844.

Eng, J.A., Frosch, M.P., Choi, K., et al., 2004. Clinical manifestations of cerebral amyloid angiopathy-related inflammation. Ann. Neurol. 55 (2), 250-256.

Greenberg, S.M., Charidimou, A., 2018. Diagnosis of cerebral amyloid Angiopathy: evolution of the Boston criteria. Stroke 49 (2), 491-497.

Kinnecom, C., Lev, M.H., Wendell, L., et al., 2007. Course of cerebral amyloid angiopathy-related inflammation. Neurology 68, 1411-1416.

Kirshner, H.S., Bradshaw, M., 2015. The inflammatory form of cerebral amyloid angiopathy or "cerebral amyloid angiopathy-related inflammation" (CAARI). Curr. Neurol. Neurosci. Rep. 15, 54.

Moussaddy, A., Levy, A., Strbian, D., et al., 2015. Inflammatory cerebral amyloid angiopathy, amyloid- $\beta$-related angiitis, and primary angiitis of the central nervous system: similarities and differences. Stroke. 46 (9), e210-e213.

Ng, D.W., Magaki, S., Terashima, K.H., et al., 2017. Amyloid- $\beta$-related angiitis: a report of 2 cases with unusual presentations. Hum. Pathol. 64, 191-197.

Piazza, F., Winblad, B., 2016. Amyloid-related imaging abnormalities (ARIA) in immunotherapy trials for Alzheimer's disease: need for prognostic biomarkers? J. Alzheimers Dis. 52 (2), 417-420.

Piazza, F., Greenberg, S.M., Savoiardo, M., et al., 2013. Anti-amyloid ?? autoantibodies in cerebral amyloid angiopathy-related inflammation: implications for amyloid-modifying therapies. Ann. Neurol. 73, 449-458.

Sakaguchi, H., Ueda, A., Kosaka, T., et al., 2011. Cerebral amyloid angiopathy-related inflammation presenting with steroid- responsive higher brain dysfunction: case report and review of the literature. J. Neuroinflammation 8, 116.

Salvarani, C., Morris, J.M., Giannini, C., et al., 2016. Imaging findings of cerebral amyloid angiopathy, A $\beta$-related angiitis (ABRA), and cerebral amyloid angiopathy-related inflammation: a single-institution 25-year experience. Medicine (Baltimore) 95 (20), e3613.

Wermer, M.J.H., Greenberg, S.M., 2018. The growing clinical spectrum of cerebral amyloid angiopathy. Curr. Opin. Neurol. 31 (1), 28-35. 